\title{
Improving Availability of Pediatric Formulations
}

\author{
Sandeep B. Bavdekar ${ }^{1}$ (D)
}

Received: 13 January 2021 / Accepted: 15 January 2021 / Published online: 28 January 2021

(C) Dr. K C Chaudhuri Foundation 2021

Based on their age and developmental abilities, children require different types of formulations. This aspect is mainly discussed in the context of oral formulations such as suitable solid (for example, smaller-sized tablets, chewable tablets, dispersible tablets and powders and granules that can be mixed with food) and liquid (for example, drops, syrups, and suspensions) formulations. However, specific pediatric aspects have to be considered even for medicines to be administered by other routes. For example, the size of a suppository should be based on the age and size of the child who is to receive the suppository. Similarly, newborn babies are vulnerable to volume overload. Hence, the volume of the drug that will have to be injected intravenously for adequate dosing has to be given due consideration. Appropriate pediatric formulations help ensure that children, especially infants and young children, receive the prescribed dose. This is an important step for making sure that children receive efficacious and safe therapy. These formulations also reduce the difficulties that parents and healthcare providers face while administering medicines to children.

However, there are a lot of hurdles in ensuring availability of the right formulations. First of all, the market for pediatric drugs is small as compared to the market for drugs required by adults. The market for pediatric-specific formulations is even smaller, since these are mostly required by children up to the age of $5 \mathrm{y}$. The pharmaceutical industry, therefore, is not overly enthusiastic about the issue. Secondly, several factors such as solubility of the principal ingredient, choice of excipients and the taste, aroma and palatability of the oral liquid formulations need to be considered [1]. This translates into investing greater time, effort, and money. The liquid formulations require greater space for storage, too.

Sandeep B. Bavdekar

sandeep.bavdekar@gmail.com

1 Department of Pediatrics, Surya Children's Hospital, Mumbai 400054, India
Nonavailability of appropriate formulations for children is a reality in various countries $[2,3]$. It is not receiving the attention it deserves. The study by Roy et al. [4] brings important issues pertaining to availability of pediatric formulations into focus. They have noted that medicines used in the treatment of mental, behavioral and rheumatologic disorders in children and those used in palliative care and neonatal care are under-represented in various pediatric essential medicines lists (EMLs). They have also flagged the issue of nonsynchronous nature of World Health Organization (WHO), Indian Academy of Pediatrics (IAP) and state EMLs for children. Medicines that are not listed in the EMLs are generally not procured on priority basis. The authors have stated that nonavailability of medicines in the market despite being listed in EMLs, and lack of demand from clinicians for specific and appropriate formulations contribute to their nonavailability. The study is limited by presentation of old (over $5 \mathrm{y}$ ) data and its generalizability is questionable, as it is a single-center study. Despite these limitations, the authors need to be commended for focusing on this important topic.

Much more needs to be done: Researchers should generate more representative data regarding availability of suitable formulations from different parts of the country, so that professional organizations and regulators can take corrective steps. These studies should include a more detailed analysis to determine the exact cause of nonavailability. Site-/region-specific factors such as resource crunch, inefficiencies in procurement processes, deficient inventory management, and limited storage space can adversely affect availability of formulations. Determination of specific reason behind nonavailability will dictate effective remedial actions. A particular formulation may not be procured, as it is not mentioned in the state's pediatric EML. Expert groups, government departments, and professional bodies should update and harmonize EMLs for children by following uniform criteria and make them more "child-consumer friendly". They should also ensure that medicines required by various pediatric sub-specialties are adequately represented in the pediatric EMLs. The importance of preparing broad-based pediatric EMLs cannot be 
overemphasized, as many health organizations and public hospitals look at EMLs for guidance while deciding about the list of medicines to be procured.

As stated earlier, many a times suitable formulations for infants and children are not developed for want of sufficient demand. Regulator-initiated steps to encourage, coax and/or compel pharmaceutical industry to generate pediatric-specific information for new drugs has shown encouraging results over the past few years. It has ensured that children are not deprived of newly developed drugs. Regulators, professional bodies, and advocacy groups need to decide if a similar strategy can be employed for augmenting the availability of appropriate formulations and ensure that any drug that is indicated in infants and young children, should be available as a suitable formulation.

\section{Compliance with Ethical Standards}

Conflict of Interest None.

\section{References}

1. European Medicines Agency. Guideline on pharmaceutical development of medicines for paediatric use. Available at: https://www.ema. europa.eu/en/documents/scientific-guideline/guidelinepharmaceutical-development-medicines-paediatric-use_en.pdf. Accessed 9 January 2021.

2. van der Vossen A, Buljac S, Akcay K, Brugma JD, Vulto A, Hanff L. Availability of age-appropriate pediatric formulations in the Netherlands: the need in daily clinical practice remains. Eur J Hosp Pharm. Published Online First: 11 November 2019. Available at: https://ejhp.bmj.com/content/early/2019/11/11/ejhpharm-2019001977. Accessed 9 January 2021.

3. Hoppu K, Rajgnathan SS. Essential medicines for children. Arch Dis Child. 2015;100(supl. 1):s38-42.

4. Roy V, Singhal S, Tayal H, Dubey AP. Availability of pediatric formulations in public health care system in India: a case study. Indian J Pediatr. 2020. https://doi.org/10.1007/s12098-020-03220-3.

Publisher's Note Springer Nature remains neutral with regard to jurisdictional claims in published maps and institutional affiliations. 